ANNALES

POLONICI MATHEMATICI

93.1 (2008)

\title{
Bundle functors on all foliated manifold morphisms have locally finite order
}

\author{
by J. Kurek (Lublin) and W. M. MikuLski (Kraków)
}

\begin{abstract}
We prove that any bundle functor $F: \mathcal{F} o l \rightarrow \mathcal{F} \mathcal{M}$ on the category $\mathcal{F}$ ol of all foliated manifolds without singularities and all leaf respecting maps is of locally finite order.
\end{abstract}

Let $\mathcal{M} f_{m}$ be the category of $m$-dimensional manifolds and their embeddings and $\mathcal{F} \mathcal{M}$ be the category of all fibred manifolds and their fibred maps. In [9], R. Palais and C. Terng showed that any natural bundle in the sense of A. Nijenhuis [8] (bundle functor) $F: \mathcal{M} f_{m} \rightarrow \mathcal{F M}$ has finite order $\operatorname{ord}(F) \leq 2^{f}+1$, where $f=\operatorname{dim}\left(F_{0} \mathbb{R}^{m}\right)$. (We remark that a bundle functor $F: \mathcal{M} f_{m} \rightarrow \mathcal{F} \mathcal{M}$ is of order $r$ if for any $\mathcal{M} f_{m}$-maps $\varphi, \psi: M \rightarrow N$ and any $x \in M$, from $j_{x}^{r} \varphi=j_{x}^{r} \psi$ it follows that $F \varphi=F \psi$ on the fiber of $F M$ over $x$.) In [1], D. Epstein and W. Thurston showed that $\operatorname{ord}(F) \leq 2 f+1$. In [11], A. Zajtz presented the best inequality

$$
\operatorname{ord}(F) \leq \max \left(\frac{f}{m-1}, \frac{f}{m}+1\right)
$$

if $m>1$. In [2], I. Kolář, P. Michor and J. Slovák extended the result from [11] to bundle functors $F: \mathcal{F} \mathcal{M}_{m, n} \rightarrow \mathcal{F} \mathcal{M}$, where $\mathcal{F} \mathcal{M}_{m, n}$ is the category of fibred manifolds with $m$-dimensional bases and $n$-dimensional fibers and their fibred embeddings, and obtained the estimate ord $(F) \leq 2 f+1$ for all $m, n$, and

$$
\operatorname{ord}(F) \leq \max \left(\frac{f}{m-1}, \frac{f}{m}+1, \frac{f}{n-1}, \frac{f}{n}+1\right)
$$

if $m>1$ and $n>1$, where $f=\operatorname{dim}\left(F_{(0,0)}\left(\mathbb{R}^{m} \times \mathbb{R}^{n}\right)\right.$ ) (the definition of the order of bundle functors on $\mathcal{F M}_{m, n}$ is a direct generalization of the one for bundle functors on $\left.\mathcal{M} f_{m}\right)$. From [2] it follows that every product preserving bundle functor $F: \mathcal{M} f \rightarrow \mathcal{F M}$, where $\mathcal{M} f$ is the category of all

2000 Mathematics Subject Classification: 58A05, 58A20, 58A32.

Key words and phrases: foliated manifold, bundle functor, order of bundle functor. 
manifolds and all maps, is of finite order $\operatorname{ord}(F)=\operatorname{ord}\left(F \mid \mathcal{M} f_{1}\right)$. In [6], the second author presented an example of a vector bundle functor $\mathcal{M} f \rightarrow \mathcal{V B}$ of strictly infinite order.

EXAmple $1([6])$. We recall that $T^{(r)} M=\left(J^{r}(M, \mathbb{R})_{0}\right)^{*}$ denotes the $r$ th order vector tangent bundle of a manifold $M$. Let $d_{r}=\operatorname{dim}\left(T_{0}^{(r)} \mathbb{R}^{r}\right)$. We set $G M=\bigoplus_{k=1}^{\infty} \bigwedge^{d_{k}} T^{(k)} M$. Then $G M$ is a finite-dimensional vector bundle for every manifold $M$ because for $k>\operatorname{dim}(M)$ the vector bundle $\bigwedge^{d_{k}} T^{(k)} M$ is the zero-bundle. Hence the direct sum in the definition of $G M$ is in fact a finite sum. For a mapping $f: M \rightarrow N$ the induced mapping $G f: G M \rightarrow G N$ is defined in the natural way from $T^{(k)} f: T^{(k)} M \rightarrow T^{(k)} N$. The vector bundle functor $G$ is of strictly infinite order because its restriction to the category $\mathcal{M} f_{k}$ is of order at least $k$.

In [7], the second author proved that every bundle functor $F: \mathcal{M f} \rightarrow$ $\mathcal{F M}$ has locally finite order in the following sense.

Proposition 1 ([7]). Let $F: \mathcal{M} f \rightarrow \mathcal{F M}$ be a bundle functor. Let $r_{m}:=\operatorname{ord}\left(F \mid \mathcal{M} f_{m}\right)$. For all maps $f_{1}, f_{2}: M \rightarrow N$ and $x \in M$, from $j_{x}^{r_{\operatorname{dim}(M)+1}} f_{1}=j_{x}^{r_{\operatorname{dim}(M)+1}} f_{2}$ it follows that $F f_{1}=F f_{2}$ on the fiber over $x$.

In [2], the above result is extended to bundle functors $F: \mathcal{F} \mathcal{M}_{m} \rightarrow$ $\mathcal{F M}$, where $\mathcal{F} \mathcal{M}_{m}$ is the category of fibred manifolds with $m$-dimensional bases and their fibred maps covering embeddings. Namely, the following proposition is proved.

Proposition $2([2])$. Let $F: \mathcal{F} \mathcal{M}_{m} \rightarrow \mathcal{F M}$ be a bundle functor. Let $r_{n}=\operatorname{ord}\left(F \mid \mathcal{F} \mathcal{M}_{m, n}\right)$. For all $\mathcal{F} \mathcal{M}_{m}$-maps $f_{1}, f_{2}: Y \rightarrow Z$ and $x \in Y$, from $j_{x}^{r_{\operatorname{dim}(Y)-m+1}} f_{1}=j_{x}^{r_{\operatorname{dim}(Y)-m+1}} f_{2}$ it follows that $F f_{1}=F f_{2}$ on the fiber over $x$.

From [4] it follows that any product preserving bundle functor $F$ : $\mathcal{F} \mathcal{M} \rightarrow \mathcal{F} \mathcal{M}$ has finite order $\operatorname{ord}(F)=\operatorname{ord}\left(F \mid \mathcal{F} \mathcal{M}_{1,1}\right)$. In [3], a fiberproduct preserving bundle functor $F: \mathcal{F} \mathcal{M} \rightarrow \mathcal{F} \mathcal{M}$ of strictly infinite order is given. From [3] it follows that any fiber-product preserving bundle functor $F: \mathcal{F M} \rightarrow \mathcal{F} \mathcal{M}$ is of locally finite order in the following sense: for all $\mathcal{F M}$-maps $f_{1}, f_{2}: Y \rightarrow Z$ and $x \in Y$ with $Y \in \mathcal{F} \mathcal{M}_{m, n}$, from $j_{x}^{r_{m}} f_{1}=$ $j_{x}^{r_{m}} f_{2}$ it follows that $F f_{1}=F f_{2}$ over $x$, where $r_{m}=\max \left(\operatorname{ord}\left(F \mid \mathcal{F} \mathcal{M}_{m, 0}\right)\right.$, $\left.\operatorname{ord}\left(F \mid \mathcal{F} \mathcal{M}_{m, 1}\right)\right)$. So, we have the following natural question.

Question 1 . Is any bundle functor $F: \mathcal{F M} \rightarrow \mathcal{F M}$ of locally finite order?

In this paper we give an affirmative answer to the above question. Since the category $\mathcal{F} \mathcal{M}$ has the same skeleton as the category $\mathcal{F}_{\text {ol }}$ of all foliated manifolds without singularities and all leaf respecting maps, it is sufficient to study the order of bundle functors on $\mathcal{F}_{\text {ol }}$. 
We recall (see [2]) that a bundle functor on $\mathcal{F}_{0} l$ is a covariant functor $F: \mathcal{F}_{o l} \rightarrow \mathcal{F} \mathcal{M}$ satisfying:

(i) (Base preservation) $B_{\mathcal{F} \mathcal{M}} \circ F=B_{\mathcal{F} \text { ol }}$, where $B_{\mathcal{F} \mathcal{M}}: \mathcal{F} \mathcal{M} \rightarrow \mathcal{M} f$ is the base functor and $B_{\mathcal{F}_{o l}}: \mathcal{F}_{o l} \rightarrow \mathcal{M f}$ is the functor $(M, \mathcal{F}) \rightarrow$ $M$. Hence the induced projections form a natural transformation $\pi: F \rightarrow B_{\mathcal{F}_{0 l} l}$.

(ii) (Localization) For every inclusion $i_{(U, \mathcal{F} \mid U)}:(U, \mathcal{F} \mid U) \rightarrow(M, \mathcal{F})$ of an open subset, $F(U, \mathcal{F} \mid U)$ is the restriction $\pi^{-1}(U)$ of $\pi: F(M, \mathcal{F})$ $\rightarrow M$ over $U$ and $F i_{(U, \mathcal{F} \mid U)}$ is the inclusion $\pi^{-1}(U) \rightarrow F(M, \mathcal{F})$.

(iii) (Regularity) $F$ transforms smoothly parametrized families of $\mathcal{F}_{o l}$ maps into smoothly parametrized families of fibred maps.

EXAMPLE 2. A well-known example of a bundle functor $F: \mathcal{F}$ ol $\rightarrow \mathcal{F M}$ is the normal bundle functor $N: \mathcal{F}$ ol $\rightarrow \mathcal{F M}$ transforming any foliated manifold $(M, \mathcal{F})$ into its normal bundle $N(M, \mathcal{F})=T M / T \mathcal{F}$ and any $\mathcal{F}$ olmap $f:(M, \mathcal{F}) \rightarrow\left(M_{1}, \mathcal{F}_{1}\right)$ into the quotient map $N f=[T f]: N(M, \mathcal{F}) \rightarrow$ $N\left(M_{1}, \mathcal{F}_{1}\right)$. This bundle functor $N$ is product preserving. Another product preserving bundle functor $\mathcal{F}_{o l} \rightarrow \mathcal{F} \mathcal{M}$ can be found in [10]. (In [5], the second author described all product preserving bundle functors $F: \mathcal{F}_{o l} \rightarrow$ $F \mathcal{M}$ in terms of Weil algebra homomorphisms $\mu: A \rightarrow B$.)

EXAMPLE 3. Let $F=T \otimes V: F \mathcal{M} \rightarrow \mathcal{V B}$ be the vector bundle functor sending any fibred manifold $p: Y \rightarrow M$ into the tensor product $F Y=$ $T M \otimes_{Y} V Y$ of the tangent bundle $T M$ with the vertical bundle $V Y \rightarrow Y$ of $Y \rightarrow M$, and any $\mathcal{F M}$-map $f: Y \rightarrow Y_{1}$ covering $f: M \rightarrow M_{1}$ into $F f=$ $T f \otimes V f: F Y \rightarrow F Y_{1}$. This bundle functor $F$ is fibre product preserving but is not product preserving. Using the standard "gluing" argument one can uniquely extend $F$ to $\widetilde{F}: \mathcal{F}$ ol $\rightarrow \mathcal{F M}$. In this way we obtain a vector bundle functor which is not product preserving. (In [3], I. Kolár and the second author described all fibre product preserving vector bundle functors $F: \mathcal{F M} \rightarrow \mathcal{V B}$. The functors are of the form $F=G \otimes V: \mathcal{F} \mathcal{M} \rightarrow \mathcal{V B}$ $\left(F Y=G M \otimes_{Y} V Y, F f=G f \otimes V f\right)$ for some vector bundle functor $G$ : $\mathcal{M} f \rightarrow \mathcal{V B}$. Taking $G$ of strictly infinite order (see Example 1), we produce $F: \mathcal{F M} \rightarrow \mathcal{F M}$ of strictly infinite order. Then using the standard "gluing" argument we produce $\widetilde{F}: \mathcal{F}$ ol $\rightarrow \mathcal{F} \mathcal{M}$ of strictly infinite order.)

EXAMPLE 4. Let $S$ be a manifold. We have a trivial bundle functor $F=\operatorname{id}_{\mathcal{F}_{o l}} \times \operatorname{id}_{S}: \mathcal{F}_{O l} \rightarrow \mathcal{F} \mathcal{M}, F(M, \mathcal{F})=M \times S, F f=f \times \operatorname{id}_{S}$. This $F$ is not a product preserving bundle functor if $S$ is not one point. If $S$ is not a vector bundle, then $F$ is not a vector bundle functor.

We recall that a bundle functor $F: \mathcal{F}_{o l} \rightarrow \mathcal{F M}$ is of locally finite order if for any $m, n$ there exists a finite number $r_{m, n}$ such that for any foliated $(m+n)$-dimensional manifold $M$ with $n$-dimensional foliation $\mathcal{F}$ and 
any $\mathcal{F}_{\text {ol-maps }} f, g:(M, \mathcal{F}) \rightarrow\left(N, \mathcal{F}_{1}\right)$ (into an arbitrary foliated manifold $\left.\left(N, \mathcal{F}_{1}\right)\right)$ and any $x \in M$, from $j_{x}^{r_{m, n}} f=j_{x}^{r_{m, n}} g$ it follows that $F f=F g$ on the fiber of $F(M, \mathcal{F})$ over $x$.

The purpose of the present note is to prove the following theorem which gives an affirmative answer to Question 1.

TheOREM 1. Any bundle functor $F: \mathcal{F}$ ol $\rightarrow \mathcal{F} \mathcal{M}$ has locally finite order in the following sense: Let $m, n$ be positive integers, $(M, \mathcal{F})$ be an $(m+n)$ dimensional foliated manifold $M$ with n-dimensional foliation $\mathcal{F}$, and $x \in$ $M$ be a point. Then for all $\mathcal{F}_{\text {ol-maps }} f_{1}, f_{2}:(M, \mathcal{F}) \rightarrow\left(M_{1}, \mathcal{F}_{1}\right)$, from $j_{x}^{r(m, n)} f_{1}=j_{x}^{r(m, n)} f_{2}$ it follows that $F f_{1}=F f_{2}$ on the fibre over $x$, where $r(m, n)=\max \left(\operatorname{ord}\left(F \mid \mathcal{F} \mathcal{M}_{m+1, n}\right), \operatorname{ord}\left(F \mid \mathcal{F} \mathcal{M}_{m, n+1}\right)\right)$.

Proof. Let $f_{1}, f_{2}:(M, \mathcal{F}) \rightarrow\left(M_{1}, \mathcal{F}_{1}\right)$ be $\mathcal{F}_{\text {ol }}$-maps such that $j_{x}^{r(m, n)} f_{1}$ $=j_{x}^{r(m, n)} f_{2}$ for some $x \in M$. We show that $F f_{1}=F f_{2}$ over $x$.

(I) First we assume that $p \geq m$ and $q \geq n$. Because of the regularity of $F$ we can assume that $d_{x} f_{1}$ is of rank $m+n$. Then by the rank theorem we can assume $(M, \mathcal{F})=\left(\mathbb{R}^{m} \times \mathbb{R}^{n},\left\{\{a\} \times \mathbb{R}^{n}\right\}_{a \in \mathbb{R}^{m}}\right), x=(0,0),\left(M_{1}, \mathcal{F}_{1}\right)=$ $\left(\mathbb{R}^{p} \times \mathbb{R}^{q},\left\{\{c\} \times \mathbb{R}^{q}\right\}_{c \in \mathbb{R}^{p}}\right), f_{1}(0,0)=f_{2}(0,0)=(0,0)$ and

$$
f_{1}(x, y)=((x, 0),(y, 0))
$$

for any $(x, y) \in \mathbb{R}^{m} \times \mathbb{R}^{n}$. Let $f_{i}(x, y)=\left(\varphi_{i}(x), \psi_{i}(x, y)\right)$ for any $(x, y) \in$ $\mathbb{R}^{m} \times \mathbb{R}^{n}, i=1,2$. Define $\mathcal{F}$ ol-maps $\Phi_{i}: \mathbb{R}^{m} \times \mathbb{R}^{n} \rightarrow \mathbb{R}^{m} \times \mathbb{R}^{q}$ by

$$
\Phi_{i}(x, y)=\left(x, \psi_{i}(x, y)\right), \quad(x, y) \in \mathbb{R}^{m} \times \mathbb{R}^{n},
$$

and $\Psi_{i}: \mathbb{R}^{m} \times \mathbb{R}^{q} \rightarrow \mathbb{R}^{p} \times \mathbb{R}^{q}$,

$$
\Psi_{i}(x, z)=\left(\varphi_{i}(x), z\right), \quad(x, z) \in \mathbb{R}^{m} \times \mathbb{R}^{q} .
$$

Then $f_{i}=\Psi_{i} \circ \Phi_{i}, i=1,2$.

Define a bundle functor $G: \mathcal{F} \mathcal{M}_{m} \rightarrow \mathcal{F M}$ by $G=F \mid \mathcal{F} \mathcal{M}_{m}$. Of course, the $\Phi_{i}$ are $\mathcal{F} \mathcal{M}_{m}$-maps and $j_{(0,0)}^{r(m, n)} \Phi_{1}=j_{(0,0)}^{r(m, n)} \Phi_{2}$. Then by Proposition 2 we have $G \Phi_{1}=G \Phi_{2}$ over $(0,0) \in \mathbb{R}^{m} \times \mathbb{R}^{n}$. So $F \Phi_{1}=F \Phi_{2}$ on the fibre $F_{(0,0)}$ of $F\left(\mathbb{R}^{m} \times \mathbb{R}^{n},\left\{\{a\} \times \mathbb{R}^{n}\right\}_{a \in \mathbb{R}^{m}}\right)$ over $(0,0) \in \mathbb{R}^{m} \times \mathbb{R}^{n}$.

Hence it remains to show that $F \Psi_{1}=F \Psi_{2}$ on $F \Phi_{1}\left(F_{(0,0)}\right)$.

We define $\mathcal{F}$ ol-maps $\widetilde{\Psi}_{i}:=\varphi_{i} \times \mathrm{id}_{\mathbb{R}^{n}}: \mathbb{R}^{m} \times \mathbb{R}^{n} \rightarrow \mathbb{R}^{p} \times \mathbb{R}^{n}$ and $I_{s}:$ $\mathbb{R}^{s} \times \mathbb{R}^{n} \rightarrow \mathbb{R}^{s} \times \mathbb{R}^{q}, I_{s}(w, y)=(w,(y, 0))$. Then $\Psi_{i} \circ I_{m}=I_{p} \circ \widetilde{\Psi}_{i}$ and $I_{m}=\Phi_{1}$. Clearly, $F I_{s}$ is an embedding because $I_{s}$ is (see [2]). Then it suffices to show that $F \widetilde{\Psi}_{1}=F \widetilde{\Psi}_{2}$ over $(0,0) \in \mathbb{R}^{m} \times \mathbb{R}^{n}$.

Define a bundle functor $H: \mathcal{M f} \rightarrow \mathcal{F M}$ by $H M=F\left(M \times \mathbb{R}^{n}\right.$, $\left.\left\{\{a\} \times \mathbb{R}^{n}\right\}_{a \in M}\right), H \varphi=F\left(\varphi \times \mathrm{id}_{\mathbb{R}^{n}}\right)$. Clearly, $j_{0}^{r(m, n)} \varphi_{1}=j_{0}^{r(m, n)} \varphi_{2}$. Then by Proposition $1, H \varphi_{1}=H \varphi_{2}$ over $0 \in \mathbb{R}^{m}$. Therefore $F \widetilde{\Psi}_{1}=F \widetilde{\Psi}_{2}$ over $(0,0) \in \mathbb{R}^{m} \times \mathbb{R}^{n}$, as well, which implies $F f_{1}=F f_{2}$ over $x \in M$ under the assumption $p \geq m$ and $q \geq n$. 
(II) Now let $p$ and $q$ be arbitrary. We may assume $(M, \mathcal{F})=\left(\mathbb{R}^{m} \times \mathbb{R}^{n}\right.$, $\left.\left\{\{a\} \times \mathbb{R}^{n}\right\}_{a \in \mathbb{R}^{m}}\right), x=(0,0),\left(M_{1}, \mathcal{F}_{1}\right)=\left(\mathbb{R}^{p} \times \mathbb{R}^{q},\left\{\{c\} \times \mathbb{R}^{q}\right\}_{c \in \mathbb{R}^{p}}\right), f_{1}(0,0)=$ $f_{2}(0,0)=(0,0)$. Let $\widetilde{p} \geq \max (m, p)$ and $\widetilde{q} \geq \max (n, q)$. Let $J: \mathbb{R}^{p} \times \mathbb{R}^{q} \rightarrow$

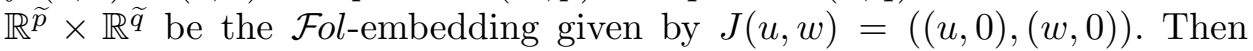
$j_{(0,0)}^{r(m, n)}\left(J \circ f_{1}\right)=j_{(0,0)}^{r(m, n)}\left(J \circ f_{2}\right)$. Hence, by (I) for $J \circ f_{i}$ instead of $f_{i}$ and $(\widetilde{p}, \widetilde{q})$ instead of $(p, q)$, we have $F\left(J \circ f_{1}\right)=F\left(J \circ f_{2}\right)$ over $(0,0) \in \mathbb{R}^{m} \times \mathbb{R}^{n}$. But $F J$ is an embedding because $J$ is. Then $F f_{1}=F f_{2}$ over $x$ as well.

From Theorem 1 we immediately obtain the following corollary.

Corollary 1. Any bundle functor $F: \mathcal{F M} \rightarrow \mathcal{F M}$ has locally finite order in the following sense: Let $m, n$ be positive integers. Let $Y \rightarrow M$ be an $\mathcal{F} \mathcal{M}_{m, n}$-object and $x \in Y$ be a point. Then for all $\mathcal{F} \mathcal{M}$-morphisms $f_{1}, f_{2}: Y \rightarrow Y_{1}$, from $j_{x}^{r(m, n)} f_{1}=j_{x}^{r(m, n)} f_{2}$ it follows that $F f_{1}=F f_{2}$ over $x$, where $r(m, n)$ is defined as in Theorem 1 .

EXAMPLE 5. Let $G: \mathcal{M} f \rightarrow \mathcal{V B}$ be the vector bundle functor of strictly infinite order as in Example 1. We define a bundle functor $F=G: \mathcal{F} o l \rightarrow$ $\mathcal{V B}, F(M, \mathcal{F})=G M, F f=G f$. This bundle functor is of strictly infinite order. It is of locally finite order, but in this case we cannot replace $r(m, n)$ in Theorem 1 by an $r(m)$ depending only on $m$ (in contrast to Example 3).

\section{References}

[1] D. B. A. Epstein and W. P. Thurston, Transformation groups and natural bundles, Proc. London Math. Soc. 38 (1979), 219-236.

[2] I. Koláŕ, P. W. Michor and J. Slovák, Natural Operations in Differential Geometry, Springer, Berlin, 1993.

[3] I. Koláŕ and W. M. Mikulski, Fiber product preserving bundle functors on all morphisms of fibered manifolds, Arch. Math. (Brno) 42 (2006), 285-293.

[4] W. M. Mikulski, Product preserving bundle functors on fibered manifolds, ibid. 32 (1996), 307-316.

[5] -, Product preserving bundles on foliated manifolds, Ann. Polon. Math. 84 (2004), $67-74$.

[6] -, There exists a prolongation functor of infinite order, Časopis Pěst. Mat. 114 (1989), 57-59.

[7] -, Natural transformations of Weil functors into bundle functors, Rend. Circ. Mat. Palermo (2) Suppl. 22 (1990), 177-191.

[8] A. Nijenhuis, Natural bundles and their general properties, in: Differential Geometry (in Honor of K. Yano), Kinokuniya, Tokyo, 1972, 317-334.

[9] R. S. Palais and C. L. Terng, Natural bundles have finite order, Topology 16 (1977), 271-277.

[10] R. A. Wolak, On transverse structures of foliations, Rend. Circ. Mat. Palermo (2) Suppl. 9 (1985), 227-243. 
[11] A. Zajtz, The sharp upper bound on the order of natural bundles of given dimensions, Bull. Soc. Math. Belg. Sér. B 39 (1987), 347-357.

Institute of Mathematics

Maria Curie-Skłodowska University

Pl. M. Curie-Skłodowskiej 1

20-031 Lublin, Poland

E-mail: kurek@hektor.umcs.lublin.pl
Institute of Mathematics

Jagiellonian University

Reymonta 4

30-059 Kraków, Poland

E-mail: Wlodzimierz.Mikulski@im.uj.edu.pl

Received 22.6.2007

and in final form 3.10.2007 